\title{
PSICOLOGÍA AMBIENTAL: UN BREVE RECORRIDO POR LA DISCIPLINA*
}

\author{
Daniel Chaustre Jota \\ https://orcid.org/0000-0002-1535-4202 \\ Universidad Católica Andrés Bello, Caracas, Venezuela \\ Correo electrónico: dchaustr@ucab.edu.ve
}

Recibido: 1 de junio del 2021 / Aceptado: 20 de agosto del 2021

doi: https://doi.org/10.26439/persona2021.n024(2).5212

\begin{abstract}
RESUMEN. El objetivo de este artículo es realizar una revisión de literatura acerca de la psicología ambiental, disciplina que estudia la relación entre el individuo y el medioambiente. Dada la gran diversidad en este campo, tomando en cuenta el ambiente social y físico, así como el ambiente natural y el ambiente construido, se elaboró la definición de la psicología ambiental y sus características más resaltantes. Posteriormente, se analizaron los métodos de estudio habitualmente empleados en la disciplina y los posibles niveles de análisis considerados en la psicología ambiental: desde microambientes hasta ambientes globales. A continuación, se comentaron dos de los campos de estudio más destacados: las actitudes ambientales y el desarrollo sustentable. Finalmente, se discute acerca del futuro de la psicología ambiental, considerando el incremento de las problemáticas y preocupaciones ambientales.
\end{abstract}

Palabras clave: psicología ambiental / revisión de literatura / desarrollo sustentable / ambiente

\footnotetext{
* Agradezco al profesor Guillermo Yáber Oltra por fomentar la elaboración del documento, así como por sus sugerencias durante todo el proceso de redacción.
} 


\section{ENVIRONMENTAL PSYCHOLOGY: A BRIEF REVIEW OF THE FIELD}

ABSTRACT. This paper aims to review the literature about environmental psychology, a field that examines the relation between the individual and the environment. Due to the diversity of approaches to the study of the individual-environment interaction, including social and physical environment as well as natural and artificial environment, a definition of environmental psychology and its main characteristics are presented. Research methods usually applied in the field, as well as levels of analysis (from microenvironment to global environments) are analyzed. Then, two of the most relevant study fields are examined: environmental attitudes and sustainable development. Finally, the future of environmental psychology is discussed, considering the raise of environmental problems and concerns.

Keywords: environmental psychology / literature review / sustainable development / environment 


\section{INTRODUCCIÓN}

¿Qué estudia la psicología ambiental? ¿Se ocupa de los problemas del medioambiente como el calentamiento global, la deforestación y la contaminación de los mares? ¿0 se interesa por la ecología, el reciclaje y la concientización ambiental? En cierta medida, la psicología ambiental está interesada en estos temas, pero no de la misma manera que un activista o un político. Un aspecto clave es que se está hablando de psicología ambiental, y es este término, a veces tan presto a concepciones erróneas, el que le da un matiz particular a esta disciplina.

Las concepciones erróneas (misconceptions, en inglés) abundan en muchas áreas del conocimiento. La psicología es una de las disciplinas científicas donde estas concepciones parecen proliferar en mayor medida, lo que afecta no solo a quienes se encuentran fuera de la profesión, sino también a estudiantes y profesionales (Bensley \& Lillienfield, 2017). Si se toma en cuenta que los aspectos ambientales, como el cambio climático, también están plagados de concepciones erróneas (Dutt \& González, 2012), la intersección entre ambos tópicos requiere de especial precisión para disipar las ideas erróneas que giran en torno a ellos.

Lo que en la actualidad se denomina psicología ambiental se consolidó como una disciplina científica en la década de 1960 en Estados Unidos, aunque tuvo precursores en distintos países, como Alemania, Japón y Canadá (Gifford, 2016). Desde entonces la disciplina ha podido alcanzar la internacionalización, estando presente en diversos países. El interés por el estudio del papel del medioambiente como una influencia sobre las personas se ha incrementado de la mano del surgimiento de diversas problemáticas cada vez más relevantes: en el mundo, la contaminación y el calentamiento global crecen constantemente como problemas urgentes e inmediatos; en el ámbito local, la falta de alimentos y de agua son problemas que atentan contra la calidad de vida de muchas personas al no poder satisfacer sus necesidades básicas (Fleury-Bahi et al., 2017). Estas situaciones adquirieron protagonismo en diversos medios durante los años sesenta, coincidiendo con el surgimiento de la psicología ambiental, y permanecen como problemas sin resolver.

La psicología ambiental no solo se centra en estudiar el efecto que el medioambiente físico tiene sobre las personas, sino la manera en que las personas afectan, de forma perjudicial o beneficiosa, a su medioambiente. Al ser esta una relación recíproca, la psicología ambiental cumple un papel principal al momento de determinar las mejores prácticas para sacar provecho de los recursos del medioambiente, de una forma no perjudicial a largo plazo; así, surge el término desarrollo sustentable, a través del cual se recalca que el desarrollo puede llevarse a cabo de una manera en que no se agoten los recursos del planeta (Bechtel, 2009). 
Tomando esto como punto de partida, la disciplina sirve como un mediador entre las necesidades individuales y las necesidades sociales, pero no solo de los grupos cercanos en tiempo y espacio, sino también de futuras generaciones. Permite, entonces, ampliar el horizonte de los problemas que enfrentan las personas en la actualidad, pues plantea que las posibles respuestas que muestren resultados positivos a corto plazo no siempre son las soluciones que utilizan los recursos de manera más óptima a largo plazo.

A pesar de estos prometedores objetivos, una de las grandes limitantes que ha presentado la psicología ambiental radica en la amplitud de su campo de estudio. Considerada por necesidad como un campo interdisciplinario, debido a la cantidad de fuentes diversas que explicarían la relación entre individuo y medioambiente (Bechtel, 2009), puede ser complicado para el novel que ingresa en el campo de la psicología ambiental hacerse una idea de los límites y del foco de estudio de la disciplina.

Es necesario, pues, delimitar en qué consiste la psicología ambiental, cuáles son sus métodos, así como los problemas principales en los que múltiples profesionales centran su atención, y cuáles son algunos de los tópicos de estudio en la actualidad. Es el objetivo de esta revisión brindarle al lector una idea general de la psicología ambiental como una disciplina científica, y así esclarecer las concepciones erróneas que suelen estar vinculadas a ella.

\section{DEFINICIÓN DE PSICOLOGÍA AMBIENTAL}

La psicología ambiental es una disciplina reciente, la cual se define de formas relativamente sencillas, pero que en su sencillez resultan bastante amplias; esto último complejiza enormemente la tarea de resumir cuál es el alcance de la disciplina, ya que su foco de interés es sumamente extenso. Este problema, originado en principio por el interés de diversos autores por no restringir el desarrollo de la incipiente disciplina al dar una definición que limite el campo de estudio, se mantiene vigente en el sentido de la heterogeneidad de los temas que son abordados (Aragonés \& Amérigo, 1998).

La psicología ambiental ha sido definida como la disciplina que estudia la relación entre los individuos y el ambiente: natural y construido (Steg et al., 2019). En este sentido, el interés principal radica en analizar las distintas maneras en que el individuo se ve afectado y afecta a su medioambiente. Aragonés y Amérigo (1998) resaltan que la psicología ambiental se centra en el estudio de las relaciones recíprocas entre la conducta y el medioambiente social y físico, ya sea natural o creado por el hombre. De esta forma, el término ambiente es entendido de manera global, abarcando la dimensión social y física, así como el ambiente construido por el ser humano y el ambiente natural. Esta idea contrasta con la concepción común de que el ambiente se refiere únicamente al ambiente natural. 
Otros autores han hecho hincapié en cuáles son los tópicos particulares de estudio, en su intento por brindar una definición más precisa. Baldi y García (2006) plantean que la psicología ambiental es una rama científica que "trata acerca del comportamiento humano y su relación con diversos problemas y eventos ambientales" (p. 159). Agregan que, entre otros aspectos, "aborda el estudio de los factores psicológicos, como creencias, actitudes, competencias, motivos, conocimientos y creencias ambientales, y la manera en la cual estas variables afectan y son afectadas por la interacción individuomedioambiente" (p. 159). Por su parte, Steg et al. (2019) señalan que el interés principal se centra en examinar la influencia del ambiente en las experiencias, las conductas y el bienestar humano, así como la influencia de los humanos sobre el ambiente, considerando los factores de la conducta ambiental y tratando de promover conductas proambientales. Así, para estos autores, existe un marcado interés por la intervención entre los principales tópicos de estudio.

Como se puede apreciar, las definiciones anteriores convergen en ciertos aspectos clave. Lo que Aragonés y Amérigo (1998), así como Steg et al. (2019), han denominado las características de la psicología ambiental resumen de cierta manera los elementos comunes que definen a la disciplina:

1. Se estudian las relaciones entre la conducta y el medioambiente, considerando a este último desde una visión holística. Se brinda especial atención a la relación entre los elementos como unidades de análisis y, en menor medida, a los componentes aislados.

2. Se toman en cuenta todas las posibles relaciones entre el medioambiente y la conducta, es decir: cómo la conducta influye sobre el medioambiente, cómo este influye sobre la conducta, y cómo se producen procesos interactivos entre ambos componentes.

3. Aunque se originó a raíz de intereses marcadamente aplicados, el desarrollo de conceptos y de relaciones teóricas entre ellos ha permitido a la psicología ambiental establecerse como una disciplina básica; sin embargo, hay una estrecha relación entre teoría y aplicación para la resolución de problemas que sigue marcando los avances en la investigación. Una de las más importantes razones para el desarrollo de teorías es que permitan identificar las soluciones más efectivas a los problemas prácticos.

4. Tiene un carácter interdisciplinario. Al centrarse en la relación entre conducta y medioambiente, convergen muchas teorías y conceptos de distintas disciplinas, como la ecología, la sociología, la arquitectura y el urbanismo, entre otras. Estas proveen perspectivas particulares del fenómeno y, en conjunto, generan una visión más general de los fenómenos que se abordan. 
5. Gran parte de los estudios tienen como objetivos subyacentes la mejora de la calidad de vida y el medioambiente. Se observa un incremento en la literatura orientada a estudiar la relación entre el bienestar, la calidad de vida y el ambiente (véase Fleury-Bahi et al., 2017).

6. La naturaleza de los métodos de investigación es ecléctica, es decir que muestra una diversidad metodológica que busca ajustarse en cada caso a las condiciones de estudio: recurre a la investigación cuantitativa y cualitativa, en ambientales naturales o de laboratorio. En situaciones ideales, se busca replicar los resultados relacionados con un fenómeno particular mediante distintos diseños de investigación.

Este último punto merece especial atención. Ya que la disciplina aborda una amplia variedad de temas, sus métodos de investigación son, en consonancia, diversos. De hecho, "a través de una revisión de los principales métodos, puede concluirse que los empleados en psicología ambiental son equivalentes a los de la psicología, en general" (Aragonés \& Amérigo, 1998, p. 37).

\section{MÉTODOS DE LA PSICOLOGÍA AMBIENTAL}

Profundizar en los métodos de la psicología ambiental implica describir, a su vez, los métodos de la psicología, lo cual escapa de los límites de esta revisión debido a su gran extensión y complejidad. A pesar de esto, se puede trazar un esquema general de la investigación en psicología ambiental. El criterio de agrupación puede hacerse en función del nivel de control y participación que tenga el investigador (véase Aragonés \& Amérigo, 1998), por la técnica de investigación particular (véase Gifford, 2016) o por el tipo de ambiente donde se produce la investigación (véase Steg et al., 2019). Como puede apreciarse, no solo el campo de estudio de la psicología ambiental es variado, también lo es la manera en que se conceptualizan sus métodos de investigación.

Entre las diversas opciones, la clasificación de Steg et al. (2019), centrada en agrupar los métodos de investigación en función de las características del ambiente donde son empleados y la relación de las técnicas con estos, resulta especialmente pertinente por su relevancia en la psicología ambiental. Estos autores proponen los siguientes tipos de investigación: (a) investigación en contextos independientes del ambiente, (b) investigación en contextos artificiales y (c) investigación en contextos "reales" o naturales.

En primer lugar, las investigaciones que se producen de manera independiente del ambiente son aquellas que no necesariamente se llevan a cabo en el ambiente particular de estudio; es decir, pueden realizarse en cualquier contexto, aunque el interés se centre en recoger información sobre un ambiente particular. La técnica más utilizada en este tipo de investigaciones son las encuestas o cuestionarios; sin embargo, a pesar de que 
los autores no las consideren, también las investigaciones basadas en datos de archivos se pueden incluir en este grupo (Aragonés \& Amérigo, 1998). En ambos casos, el investigador toma un rol más pasivo, en el sentido de que su labor principal es la de recoger datos, sin intervenir directamente para generarlos. En este tipo de investigaciones se establecen relaciones entre dos o más variables; los casos en los que solo se trabaja con una se consideran de carácter descriptivo. Entre las dificultades, se encuentra que la dirección de la relación no puede determinarse con claridad, así como no se puede descartar la existencia de una tercera variable que explique la relación entre las variables de estudio. A pesar de ello, la investigación por cuestionarios permite analizar características individuales y ambientales de una manera eficiente. Una modalidad que emergió con internet son los cuestionarios en línea, los cuales son particularmente útiles para reducir costos, acceder a grandes grupos de la población y en cierta medida disminuir los efectos de la deseabilidad social (Eiroá et al., 2008).

En segundo lugar, las investigaciones realizadas en contextos artificiales son aqueIlas en las cuales se modifican elementos o aspectos, ya sean del individuo o del contexto, para determinar sus posibles consecuencias. Por tanto, el grado de manipulación hace que el contexto no sea completamente natural. Entre estas investigaciones se encuentran los experimentos de laboratorio, los experimentos de campo y las simulaciones. Los experimentos de laboratorio se producen en ambientes altamente controlados, donde se busca reducir al mínimo las posibles explicaciones alternativas de las variaciones que se produzcan en las variables de interés. Su fortaleza reside en la validez interna, es decir que las variables identificadas como causas efectivamente son aquellas que producen las variaciones en otras variables. En cambio, la validez externa, o la posibilidad de generalizar los resultados de la investigación a otros contextos, es más limitada debido a la artificialidad producto del control.

Para los experimentos de campo, la situación es distinta. En estos no se tiene el mismo grado de control que en un laboratorio, ya que se producen en contextos naturales y se incluyen manipulaciones o alteraciones en las variables independientes, esperando observar su efecto sobre las variables dependientes. Aquí no se pueden controlar todas las posibles explicaciones alternativas para los resultados observados, pero la capacidad de generalización es mayor, debido a que se trabaja en entornos naturales.

Por su parte, los estudios por simulación han existido desde hace décadas (véase McKechnie, 1977), pero debido a los avances tecnológicos han adquirido un mayor uso recientemente. A través de modelos matemáticos y de programas computacionales se pueden generar situaciones experimentales (Sánchez et al., 2012) y poner a prueba diversas reacciones. En el campo de la psicología ambiental, las simulaciones permiten generar situaciones completamente artificiales, cubriendo aspectos visuales y en ocasiones también auditivos, para determinar cómo estos pueden generar cambios en conductas, percepciones y afectos (véase Gatersleben \& Andrews, 2013). 
Finalmente, las investigaciones realizadas en contextos naturales son aquellas donde el investigador no modifica o no interviene de manera activa, limitándose a registrar y a observar lo que se presenta en un ambiente particular. Estas investigaciones pueden ser estudios de casos, y a pesar de que no son considerados por los autores originales, también los estudios observacionales pueden ser incluidos. Los estudios observacionales se consideran el paso inicial de todo proceso de investigación; lejos de limitarse a una observación ingenua de los individuos y de las características del ambiente, la investigación observacional constituye una primera aproximación y descripción del fenómeno de estudio (Sussman, 2016). Los estudios de caso, en cambio, se centran en profundizar en un tópico particular, usualmente a través de una persona, un grupo de personas o una organización, sin la pretensión de modificar o alterar el fenómeno de interés. Por lo general, las técnicas utilizadas en estos casos son de carácter cualitativo (Montero \& León, 2004).

Como se comentó anteriormente, en la psicología ambiental se emplean diversas metodologías para estudiar los fenómenos de interés, siendo una situación ideal la de poder comprobar un mismo resultado con distintas aproximaciones. La elección de un método no debe estar guiada solo por facilidades prácticas, sino también por la manera en que es más propenso a brindar la información más relevante para el investigador (Giuliani \& Scopelliti, 2009). Esto no es siempre posible, ya sea por limitaciones de carácter práctico, o por limitaciones asociadas al fenómeno específico. Por tanto, es necesario considerar tanto los elementos particulares de los contextos de estudio como el nivel de análisis que se lleve a cabo.

Debido a la amplitud de fenómenos que conciernen a la disciplina, segmentarlos en función de distintas dimensiones sociales y físicas permite un abordaje más comprensivo. A continuación, se profundizará en el tópico de los niveles de análisis en la psicología ambiental.

\section{NIVELES DE ANÁLISIS EN LA PSICOLOGÍA AMBIENTAL}

Una de las razones por las que el novel que se introduce en el campo de la psicología ambiental encuentra una gran diversidad de tópicos de estudio es que existen distintos niveles espaciales y sociales que son de interés en la disciplina. Debido a esto, se ha llegado incluso a considerar a la psicología ambiental como la psicología del espacio (Moser, 2003). Los modos en que el ambiente y los individuos interactúan pueden comprenderse de distintas maneras, pero un factor que ha de tomarse en cuenta son los aspectos del ambiente que se están estudiando; por ejemplo: ¿la investigación se centra en las características del hogar o la oficina? ¿ 0 acaso en el ambiente de un país o de un continente? La misma situación se presenta del lado de los grupos sociales: ¿se está estudiando a un sujeto aislado en un ambiente reducido? 0 , en cambio, ¿se habla de 
un individuo inmerso en un grupo de sujetos en un ambiente más amplio? La manera en que se producen y se estudian estas relaciones no son las mismas; si bien puede haber principios comunes, operan en distintos niveles $y$, por tanto, tener claridad en el nivel que se está estudiando es fundamental.

Moser (2003) plantea un esquema jerárquico que permite entender cómo se pueden agrupar las características espaciales del ambiente, considerando, además, la dimensión social. Este esquema comprende cuatro niveles: microambiente, ambiente de proximidad, ambiente público y ambiente global (véase la tabla 1).

Los microambientes (nivel I) corresponden a espacios reducidos, donde el individuo tiene un control extendido o total sobre su medioambiente, debido a que este no es compartido con otras personas, y si lo es, el número es reducido. Este nivel se ve reflejado en contextos como habitaciones $u$ hogares, y espacios de trabajo particulares.

Los ambientes de proximidad (nivel II) incluyen espacios compartidos con otras personas, donde la interacción se produce mayormente de uno a uno o en pequeños grupos. Estos ambientes son, por ejemplo, habitaciones u hogares compartidos con otras personas, así como espacios laborales donde se interactúa con otros individuos. En estos ambientes, el control que puede tener el individuo no es total, ya que se comparte con las demás personas que cohabitan el espacio.

Los ambientes públicos (nivel III) presentan un control individual mediatizado, es decir, controlado o limitado por reglas preestablecidas correspondientes a los espacios particulares. Ejemplos de estos ambientes son las ciudades y los pueblos. La interacción social se convierte en comunitaria; si bien pueden surgir interacciones uno a uno o en grupos, una multiplicidad de personas se encuentra incluida en estos espacios más extensos.

Por último, tenemos el ambiente global (nivel IV), que es el más amplio y general de todos. Incluye tanto el ambiente construido como el ambiente social que forman parte de un país, e incluso el planeta. En estos casos, se considera que el control individual es casi nulo o ausente en su totalidad. 
Tabla 1

Niveles de análisis en psicología ambiental según Moser (2003)

\begin{tabular}{|c|c|c|c|}
\hline Tipo de ambiente & Dimensión social & Grado de control & Ejemplo \\
\hline $\begin{array}{l}\text { Nivel I. } \\
\text { Microambiente }\end{array}$ & Individual & Control extendido & $\begin{array}{l}\text { Espacios privados: } \\
\text { habitación particular, } \\
\text { espacio de trabajo }\end{array}$ \\
\hline $\begin{array}{l}\text { Nivel II. } \\
\text { Ambiente de } \\
\text { proximidad }\end{array}$ & $\begin{array}{l}\text { Interindividual: } \\
\text { familia, } \\
\text { compañeros de } \\
\text { trabajo }\end{array}$ & Control compartido & $\begin{array}{l}\text { Espacios compartidos: } \\
\text { habitación colectiva, lugar } \\
\text { de trabajo compartido }\end{array}$ \\
\hline $\begin{array}{l}\text { Nivel III. } \\
\text { Ambiente público }\end{array}$ & $\begin{array}{l}\text { Interindividual: } \\
\text { comunidad }\end{array}$ & Control mediatizado & Ciudades y pueblos \\
\hline $\begin{array}{l}\text { Nivel IV. } \\
\text { Ambiente global }\end{array}$ & Societal & Ausencia de control & País, continente, planeta \\
\hline
\end{tabular}

Elaboración propia

Como se puede observar, la psicología ambiental estudia la relación entre individuo y medioambiente en distintos planos o niveles, que implican aproximaciones diferentes por parte del profesional en el área. Los tópicos que se estudian en cada nivel pueden ser muy variados; sin embargo, existen algunos temas de estudio que han captado la atención de manera consistente, como las actitudes ambientales y el desarrollo sustentable.

\section{ACTITUDES AMBIENTALES}

Al igual que sucede con la psicología ambiental, delimitar qué constituyen las actitudes ambientales puede resultar problemático debido a su gran amplitud. En principio, pueden definirse como cualquier actitud dirigida a un objeto distinto de la persona, ya que el ambiente está constituido por esferas sociales y físicas, e incluye tanto el ambiente creado por el hombre como el natural (Aragonés \& Amérigo, 1998). Sin embargo, el concepto se ha limitado para ofrecer una definición más precisa, en la cual se establece que las actitudes ambientales son una colección de creencias, afectos e intenciones conductuales que una persona mantiene acerca de actividades y problemas ambientales (Schultz et al., 2004).

Un término relacionado, y usualmente confundido en la práctica, es el de preocupaciones ambientales. Estas se refieren al afecto, especialmente a la preocupación, asociado a los problemas ambientales (Schultz et al., 2004). Actualmente, se considera que las preocupaciones ambientales forman parte de las actitudes ambientales, por lo que se recomienda el uso de este término en la investigación; a pesar de eso, no es inusual encontrar autores que emplean ambas expresiones de manera intercambiable (McIntyre \& Milfont, 2015), lo cual puede contribuir a la ambigüedad asociada al término. 
En la investigación, el principal interés por estudiar las actitudes ambientales reside en determinar la influencia que estas tienen sobre las conductas proambientales. A pesar de esto, la evidencia señala que las personas suelen presentar mayores niveles de preocupaciones ambientales que no se reflejan en mayores acciones beneficiosas para el ambiente (Gifford \& Sussman, 2012). Parte de esta dificultad para establecer con claridad qué relación existe entre actitudes y conductas ambientales, de acuerdo con Gifford y Sussman (2012), se debe a la falta de comunalidad en la concepción y medición del constructo.

Las actitudes ambientales, al tratarse de un constructo latente, no pueden ser medidas u observadas directamente, sino que requieren de indicadores que permitan al investigador inferir sus propiedades. De esta forma, y ante la gran variedad de conceptualizaciones distintas que se pueden encontrar para las actitudes ambientales, los investigadores optan por generar nuevas maneras de medir el constructo, usualmente a través de cuestionarios y entrevistas, en lugar de recurrir a los métodos que ya se encuentran disponibles (Milfont \& Duckitt, 2010). Esta forma ad hoc de medir las actitudes explicaría parte de la heterogeneidad encontrada en la literatura, pero adicionalmente se deben considerar otras explicaciones.

Los resultados inconsistentes en torno a las actitudes ambientales han llevado a algunos autores a considerar que estas solo permiten predecir conductas que tienen un bajo costo en cuanto a esfuerzo y recursos. En cambio, las conductas con costos altos no se pueden predecir solo a partir de actitudes (Gifford \& Sussman, 2012), sino que se deben tomar en cuenta otros factores mediadores o moderadores. El giro que ha dado la investigación en torno a las actitudes se ha centrado no solo en describir cómo las actitudes predicen ciertas conductas, sino en identificar y generar modelos explicativos considerando los distintos factores que intervienen en esta relación. Estos factores varían desde eventos recientes, experiencias durante la niñez, edad, género, nivel socioeconómico, residencia urbana o rural, religión, visión política y del mundo, valores y metas, sesgos cognitivos, sentido de control, personalidad, educación, hasta conocimiento sobre el medioambiente (Gifford \& Nilsson, 2014; Gifford \& Sussman, 2012).

Si bien las actitudes no han demostrado ser el mejor predictor de las conductas proambientales, históricamente muchas de las intervenciones y discursos ambientalistas han ido dirigidos a modificar precisamente actitudes (Feinberg \& Willer, 2013); sin embargo, no se puede desacreditar su papel dentro de la modificación de la conducta. Es importante tomar en cuenta, como señalan Gifford y Nilsson (2014), que una multiplicidad de factores individuales determina la ejecución de una conducta particular, y las actitudes ambientales son parte de ese abanico que, en conjunto, modificará la conducta proambiental. No se pretende, entonces, abandonar el estudio de las actitudes ambientales, sino enmarcarlo en un modelo conceptual que involucre a la diversidad de factores 
que permitirá alcanzar una mayor comprensión de cómo se produce la conducta. En vista del carácter marcadamente aplicado de la disciplina, comprender cómo modificar las conductas ambientales es un punto de sumo interés frente a las problemáticas actuales y futuras.

\section{DESARROLLO SUSTENTABLE}

Hace décadas se ha considerado que el medioambiente es uno de los campos interdisciplinarios que han recibido una atención cada vez más prioritaria por parte de la comunidad científica mundial (Baldi y García, 2005). A pesar de esto, en la actualidad existen muchas concepciones erróneas acerca del cambio climático (Dutt y González, 2012), el efecto invernadero y la capa de ozono (Khalid, 2001), el reciclaje (Geyer et al., 2015), y también sobre qué es el ambiente (McGuinn et al., 2012) y la sustentabilidad (Leal, 2000).

Como se mencionó anteriormente, la disciplina data de la década de 1960, pero a pesar de esto los problemas ambientales se han vuelto más acuciantes desde entonces. Lejos de estar zanjados, estos tópicos que despertaron el interés en la disciplina de la psicología ambiental han adquirido mayor relevancia. Tal como señala Moser (2003), el principal objetivo de la psicología ambiental a partir del siglo XXI se ha centrado en el desarrollo sustentable.

El desarrollo sustentable se contrapone al desarrollo económico que genera altos niveles de degradación del medioambiente; es decir, es una postura crítica ante el crecimiento económico que conduce tanto a la sobreexplotación como a la degradación de los recursos naturales (Gutiérrez, 2008). En este sentido, en el desarrollo sustentable se busca emplear los recursos necesarios para satisfacer las necesidades actuales, sin que eso repercuta en la posibilidad de que futuras generaciones no puedan hacer uso de tales recursos. Como plantea Gutiérrez (2008), el desarrollo sustentable busca alcanzar tres objetivos: (i) un desarrollo que tome en consideración la satisfacción de las generaciones actuales, (ii) un desarrollo respetuoso del medioambiente, y (iii) un desarrollo que no sacrifique los derechos de las futuras generaciones.

Alcanzar un desarrollo sustentable es una labor que requiere de una participación conjunta de distintas instituciones de la sociedad. Si bien también es necesario el involucramiento individual, diversas industrias deben modificar sus prácticas de desarrollo para poder generar cambios significativos. Esto incluye a múltiples sectores como el educativo, tanto a nivel universitario (Leal et al., 2020) como de educación básica (CantúMartínez, 2014); el turístico (Wojtarowski et al., 2016); el empresarial (Carro-Suárez et al., 2017); y especialmente el económico y el de salud (Castro, 2018). Por tanto, implementar efectivamente un desarrollo sustentable es una tarea que demanda una planificación conjunta de diferentes sectores y disciplinas para poder ser alcanzado. 


\section{FUTURO DE LA PSICOLOGÍA AMBIENTAL}

Las personas interactúan constantemente con el medioambiente, y en muchos casos se producen interpretaciones inadecuadas de las asociaciones que hay entre las conductas ambientales y sus consecuencias, ya que muchos efectos sobre el ambiente se evidencian de manera retrasada en el tiempo (Dutt \& González, 2012). Esto dificulta en gran medida que el espectador ingenuo de las problemáticas ambientales pueda relacionar directamente, a partir de su experiencia, cómo sus conductas impactan sobre su medioambiente. Esta es una de las grandes trabas que enfrenta la disciplina, ya que solventar muchas de las problemáticas ambientales requiere de una participación conjunta de individuos y de sectores de producción.

A pesar de que la psicología ambiental tiene una historia relativamente corta, la necesidad de desarrollar propuestas sustentadas en la investigación se ha vuelto cada vez más apremiante. Dado que los recursos ambientales son finitos y que gran parte del consumo de recursos por parte de los seres humanos no es sostenible a largo plazo, progresivamente es más evidente el impacto que han tenido estas prácticas sobre el medioambiente. Es necesario, entonces, articular las propuestas de la disciplina con las demás áreas, a niveles micro y macro, para alcanzar un mayor impacto y promover conductas y prácticas proambientales que devengan no solo en el beneficio de la generación actual, sino de las futuras, ya que las acciones tomadas en la actualidad solo mostrarán su impacto en el futuro.

Es necesaria, entonces, una aproximación interdisciplinaria y organizada para promover alternativas sustentables al uso de recursos y políticas públicas que incentiven la sustentabilidad de diversos sectores, además de concientizar sobre las problemáticas ambientales y desarrollar planes de acción desde niveles micro hasta niveles macroambientales, tanto a corto como a largo plazo. Finalmente, en la medida en que las problemáticas ambientales se hacen más acuciantes, y al mismo tiempo las preocupaciones ambientales tienden a aumentar y las personas acuden en busca de alternativas, será necesario que los profesionales de la psicología ambiental sean capaces de aportar, mediante las herramientas propias de la disciplina, respuestas satisfactorias.

\section{REFERENCIAS}

Aragonés, J. I., \& Amérigo, M. (1998). Psicología ambiental. Aspectos conceptuales y metodológicos. En J. I. Aragonés y M. Amérigo (Eds.), Psicología ambiental (pp. 25-42). Pirámide.

Baldi, G., \& García, E. (2005). Calidad de vida y medio ambiente. La psicología ambiental. Universidades, 30, 9-16. https://www.redalyc.org/articulo.oa?id=37303003 
Baldi, G., \& García, E. (2006). Una aproximación a la psicología ambiental. Fundamentos en Humanidades, 7(13-14), 157-168. https://www.redalyc.org/articulo. oa?id=18400708

Bechtel, R. B. (2009). Environmental Psychology. En I. B. Weiner \& W. E. Craighead (Eds.), The Corsini Encyclopedia of Psychology (p. 311: 1-3). John Willey \& Sons. https:// doi.org/10.1002/9780470479216.corpsy0311

Bensley, D. A., \& Lilienfeld, S. O. (2017). Psychological Misconceptions: Recent Scientific Advances and Unresolved Issues. Current Directions in Psychological Science, 26(4), 377-382. https://doi.org/10.1177/0963721417699026

Cantú-Martínez, P. C. (2014). Educación ambiental y la escuela como espacio educativo para la promoción de la sustentabilidad. Revista Electrónica Educare, 18(3), 39-52. http://dx.doi.org/10.15359/ree.18-3.3

Carro-Suárez, J., Sarmiento-Paredes, S., \& Rosano-Ortega, G. (2017). La cultura organizacional y su influencia en la sustentabilidad empresarial. La importancia de la cultura en la sustentabilidad empresarial. Estudios Gerenciales, 33, 352-365. https://doi.org/10.1016/j.estger.2017.11.006

Castro, A. (2018). Economía, salud, desarrollo humano e innovación en el desarrollo sustentable. Conocimiento Global, 3(1), 1-9.

Dutt, V., \& González, C. (2012). Decisions from Experience Reduce Misconceptions about Climate Change. Journal of Environmental Psychology, 32(1), 19-29. https://doi. org/10.1016/j.jenvp.2011.10.003

Eiroá, F. J., Fernández, I., \& Pérez, P. (2008). Cuestionarios psicológicos e investigación en internet: una revisión de la literatura. Anales de Psicología, 24(1), 150-157. https:// doi.org/10.6018/analesps

Feinberg, M., \& Willer, R. (2013). The Moral Roots of Environmental Attitudes. Psychological Science, 24(1), 56-62. https://doi.org/10.1177/0956797612449177

Fleury-Bahi, G., Pol, E., \& Navarro, O. (2017). Introduction: Environmental Psychology and Quality of Life. En G. Fleury-Bahi, E. Pol \& O. Navarro (Eds.), Handbook of Environmental Psychology and Quality of Life Research (pp. 1-8). Springer.

Gatersleben, B., \& Andrews, M. (2013). When Walking in Nature Is Not Restorative: The Role of Prospect and Refuge. Health \& Place, 20,91-101. https://doi.org/10.1016/j. healthplace.2013.01.001

Geyer, R., Kuczenski, B., Zink, T., \& Henderson, A. (2015). Common Misconceptions about Recycling. Journal of Industrial Ecology, 20(5) 1010-1017. https://doi.org/10.1111/ jiec.12355 
Gifford, R. (Ed.). (2016). Research Methods for Environmental Psychology. Wiley-Blackwell.

Gifford, R., \& Nilsson, A. (2014). Personal and Social Factors that Influence Pro-Environmental Concern and Behaviour: A Review. International Journal of Psychology, 49(3), 141-157. https://doi.org/10.1002/ijop.12034

Gifford, R., \& Sussman, R. (2012). Environmental Attitudes. En S. D. Clayton (Ed.), Oxford Library of Psychology. The Oxford Handbook of Environmental and Conservation Psychology (pp. 65-80). Oxford University Press. https://doi.org/10.1093/ oxfordhb/9780199733026.013.0004

Giuliani, M. V., \& Scopelliti, M. (2009). Empirical Research in Environmental Psychology: Past, Present, and Future. Journal of Environmental Psychology, 29, 375-386. https://doi.org/10.1016/j.jenvp.2008.11.008

Gutiérrez, E. (2008). De las teorías del desarrollo al desarrollo sustentable. Historia de la constitución de un enfoque multidisciplinario. Ingenierías, 21(39), 21-35.

Khalid, T. (2001). Pre-Service Teachers' Misconceptions Regarding Three Environmental Issues. Canadian Journal of Environmental Education, 6(1), 102-120. https://cjee. lakeheadu.ca/article/view/290

Leal, W. (2000). Dealing with Misconceptions on the Concept of Sustainability. International Journal of Sustainability in Higher Education, 1(1), 9-19. https://doi. org/10.1108/1467630010307066

Leal, W., Kovaleva, M., Fritzen, B., Fudjumdjum, H., Emblen-Perry, K., Platje, J., Tuladhar, L., Vasconcelos, C., LeVasseur, T. J., Minhas, A., Farinha, C. S., Buil-Fabregá, M., Novo-Corti, I., Țîrcă, D.-M., \& Da Cunha, D. A. (2020). Sustainability Practices at Private Universities: A State-of-the-Art Assessment. International Journal of Sustainable Development \& World Ecology, 27(8), 1-15. https://doi.org/10.1080/13 504509.2020 .1848940

McGuinn, L. A., Ghazarian, A. A., Ellison, G. L., Harvey, C. E., Kaefer, C. M., \& Reid, B. (2012). Cancer and Environment: Definitions and Misconceptions. Environmental Research, 112, 230-234. https://doi.org/10.1016/j.envres.2011.10.009

McIntyre, A., \& Milfont, T. L. (2015). Who Cares? Measuring Environmental Attitudes. En R. Gifford (Ed.), Research Methods for Environmental Psychology (pp. 93-114). WileyBlackwell.

McKechnie, G. E. (1977). Simulation Techniques in Environmental Psychology. En D. Stokols (Ed.), Perspectives on Environment and Behavior (pp. 169-189). Springer.

Milfont, T. L., \& Duckitt, J. (2010). The Environmental Attitudes Inventory: A Valid and Reliable Measure to Assess the Structure of Environmental Attitudes. 
Journal of Environmental Psychology, 30(1), 80-94. https://doi.org/10.1016/j. jenvp.2009.09.001

Montero, I., \& León, O. G. (2004). Sistema de clasificación del método en los informes de investigación en psicología. International Journal of Clinical and Health Psychology, 5(1), 115-127.

Moser, G. (2003). La psicología ambiental en el siglo xxI: el desafío del desarrollo sustentable. Revista de Psicología de la Universidad de Chile, 12(2), 11-17. https:// www.redalyc.org/articulo.oa?id=26412202

Sánchez, M. P., De la Garza, A., \& López, E. O. (2012). Simulaciones computacionales sobre cuestiones ambientales en dos grupos de contraste. Liberabit, 19(2), 223-233. http://www.scielo.org.pe/pdf/liber/v19n2/a08v19n2.pdf

Schultz, P. W., Shriver, C., Tabanico, J. J., \& Khazian, A. M. (2004). Implicit Connections with Nature. Journal of Environmental Psychology, 24(1), 31-42. https://doi.org/10.1016/ S0272-4944(03)00022-7

Steg, L., Van den Berg, A. E., \& De Groot, J. I. M. (2019). Environmental Psychology: History, Scope, and Methods. En L. Steg y J. I. M. de Groot (Eds.), Environmental Psychology

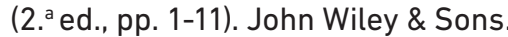

Sussman, R. (2016). Observational Methods: The First Step in Science. En R. Gifford (Ed.), Research Methods for Environmental Psychology (pp. 9-27). Wiley-Blackwell.

Wojtarowski, A., Silva, E., Piñar, M. A., \& Negrete, J. A. (2016). La responsabilidad social empresarial como pieza clave en la transición hacia el desarrollo sustentable en el sector turístico. Revista de Turismo y Patrimonio Cultural, 14(1), 127-139. https:// doi.org/10.25145/j.pasos.2016.14.008 